\title{
Design of a Qubit and a Decoder in Quantum Computing Based on a Spin Field Effect
}

\author{
A. A. Suratgar ${ }^{1}$, S. Rafiei ${ }^{* 2}$, A. A. Taherpour ${ }^{3}$, A. Babaei $^{4}$ \\ ${ }_{1}^{1}$ Assistant Professor, Electrical Engineering Department, Faculty of Engineering, Arak University, Arak, Iran. \\ ${ }^{1}$ Assistant Professor, Electrical Engineering Department, Amirkabir University of Technology, Tehran, Iran. \\ ${ }^{2}$ Young Researchers Club, Aligudarz Branch, Islamic Azad University, Aligudarz, Iran. \\ *saeid.rafiei@iau-aligudarz.ac.ir \\ ${ }^{3}$ Professor, Chemistry Department, Faculty of Science, Islamic Azad University, Arak Branch, Arak, Iran. \\ ${ }^{4}$ faculty member of Islamic Azad University, Khomein Branch, Khomein,
}

\begin{abstract}
In this paper we present a new method for designing a qubit and decoder in quantum computing based on the field effect in nuclear spin. In this method, the position of hydrogen has been studied in different external fields. The more we have different external field effects and electromagnetic radiation, the more we have different distribution ratios. Consequently, the quality of different distribution ratios has been applied to the suggested qubit and decoder model. We use the nuclear property of hydrogen in order to find a logical truth value. Computational results demonstrate the accuracy and efficiency that can be obtained with the use of these models.
\end{abstract}

Keywords: quantum computing, qubit, decoder, gyromagnetic ratio, spin.

\section{Introduction}

Up to now many papers deal with the possibility to realize a reversible computer based on the laws of quantum mechanics [1].

Modern quantum chemical methods provide powerful tools for theoretical modeling and analysis of molecular electronic structures. Implementation of quantum information processing based on spatially nuclear spins in stable molecules is one of the wide discussable applied sciences areas [2-4].

For realizing quantum computing, some physical systems, such as nuclear magnetic resonance, trapped irons, cavity quantum electrodynamics, and optical systems have been proposed. These systems have the advantage of high quantum coherence but cannot be integrated easily to form large-scale circuits. Owing to large-scale integration and relatively high quantum coherence, Josephson charge and phase qubits, based on the macroscopic quantum effects in low-capacitance Josephson junction circuits, have recently been used in quantum information processing [7]. In this paper different frequency, spin positions and different hydrogen atoms in compound applied for the qubit and decoder designing.

\section{An overview on quantum concepts}

In this chapter a short introduction is presented into the interesting field of quantum in physics, Moore's law and a summary of the quantum computer.

\subsection{Quantum in physics}

In classical physics, the physical states of an object of interest can be defined exactly to a degree, which is mainly limited by experimental factors such as random and systematic errors. The measurement of a physical state in quantum mechanics is different as it includes intrinsic uncertainty, which cannot be influenced by improvements in experimental techniques. This concept, originally proposed by Heisenberg, is called the uncertainty principle, which states that the uncertainties of measurement of energy $\Delta E$ and interval of time $\Delta t$, during which a microscopic particle possesses that energy:

$$
\Delta E . \Delta t \geq h
$$


Where $h$ is Planck's constant. Hence, only the probabilities of getting particular results can be obtained in quantum mechanical experiments, fundamentally distinguishing them from the classical ones. The uncertainties in quantummechanical measurements stem from the disturbance which measurement itself causes to the measured state at a microscopic scale. In NMR, the life times of spin states do not generally exceed the spin-lattice relaxation time $T_{1}$, and therefore the half-widths of NMR lines in spectra must be at least of the order of $1 / T_{1}[2,3,8]$.

The uncertainties featured in quantum mechanical measurements lead to a probability interpretation of phenomena, where the quantum mechanical states are described by the wave functions given in particular representation. In Dirac formulism of quantum mechanics used throughout this text, a state of a quantum mechanical system is described by the vector called ket and written as $|\psi\rangle$. The use of ket instead of the wave function allows the form of analysis, which is independent of the particular representation chosen. In this formulism, different representations are regarded as rotations in the vector space, hence the ket $|\psi\rangle$ represents the quantum state no matter what representation is chosen for the analysis [9].

The number of the ket components is $2(I)+1$, where $I$ is spin. Thus, for spin $1 / 2$ the ket has two components, each of which is a complex number. The number is represented by its real part only when the imaginary part is zero [9].

In NMR the nuclear spin magnetization is manipulated by applying a magnetic field which is (a) transverse to the static magnetic field, i.e., in the $x y$-plane, and (b) oscillating at close to the Larmor frequency of the spins. Such a field is created by passing the output of a radio-frequency transmitter through a small coil which is located close to the sample [2, 4].

If the field is applied along the $x$-direction and is oscillating at $\omega_{R F}$, the Hamiltonian for one spin is

$$
H=\omega_{0} I_{z}+2 \omega_{1} \cos \omega_{R F} t I_{X}
$$

The first term represents the interaction of the spin with the static magnetic field and the second one represents the interaction with the oscillating field. The strength of the latter is given by $\omega_{1}$. It is difficult to work with this Hamiltonian as it depends on time. However, this time dependence can be removed by changing to a rotating set of axes or a rotating frame. These axes rotate about the $z$-axis at frequency $\omega_{R F}$, and in the same sense as the Larmor precession. In such a set of axes the Larmor precession is no longer at $\omega 0$, but at $\left(\omega_{0}-\omega_{R F}\right)$; this quantity is called the offset, $\Omega$. The more important result of using the rotating frame is that the time dependence of the transverse field is removed. The details of how this comes about are beyond the scope of this paper but can be found in a number of standard texts on NMR. In the rotating frame, the Hamiltonian becomes time independent [2, 4].

$$
\begin{gathered}
H=\left(\omega_{0}-\omega_{R F}\right) I_{z}+\omega_{1} I_{X} \\
=\Omega I_{Z}+\omega_{1} I_{X}
\end{gathered}
$$

Commonly, the strength of the radiofrequency field is arranged to be much greater than typical offsets: $\omega_{1} » \Omega$. It is then permissible to ignore the offset term and so write the pulse Hamiltonian as (for pulses of either phase).

$$
H_{\text {pulse }, x}=\omega_{1} I_{x} \text { or } \quad H_{p u l s e, y}=\omega_{1} I_{y}
$$

Such pulses are described as hard or nonselective, in the sense that they affect spins over a range of offsets. Pulses with lower field strengths, $\omega_{1}$ are termed selective or soft [2, 4].

Single spin $1 / 2$ in static magnetic field acting along $z$-axis has two eigenkets: $\mid \alpha>$ (spin along the field) and $\mid \beta>$ (spin is opposite to the field) described by columns:

$$
|\alpha\rangle=\left(\begin{array}{l}
1 \\
0
\end{array}\right) \quad \text { and } \quad|\beta\rangle=\left(\begin{array}{l}
0 \\
1
\end{array}\right)
$$

In general, the state of spin $1 / 2$ may be represented by the combination of eigenkets written as the ket $\mid \psi>$ where: 


$$
\begin{aligned}
|\psi\rangle & =c_{1}|\alpha\rangle+c_{2}|\beta\rangle=c_{1}\left(\begin{array}{l}
1 \\
0
\end{array}\right)+c_{2}\left(\begin{array}{l}
0 \\
1
\end{array}\right) \\
& =\left(\begin{array}{r}
c_{1} \\
0
\end{array}\right)+\left(\begin{array}{c}
0 \\
C_{2}
\end{array}\right)=\left(\begin{array}{l}
C_{1} \\
C_{2}
\end{array}\right)
\end{aligned}
$$

$\mathrm{C}_{1}$ and $\mathrm{C}_{2}$ are complex numbers which relate to probabilities of a spin to be in the particular state, either in state $\mid \alpha>$ or in state $\mid \beta>$. The eigenkets are special kets, which are normally orthogonal and represent the states in which a quantummechanical system can be found when its state is measured. The particular eigenkets can be chosen for description of a quantum mechanical state, like a frame of reference. However, any chosen set of eigenkets must be normalized and complete, in order to be appropriate for a representation of a quantum mechanical state $[2,10,11]$.

In physics and mathematics, the Boltzmann distribution is a certain distribution function or probability measure for the distribution of the states of a system; it underpins the concept of the canonical ensemble, providing its underlying distribution. A special case of the Boltzmann distribution, used for describing the velocities of particles of a gas is the Maxwell-Boltzmann distribution. In more general mathematical settings, the Boltzmann distribution is also known as the Gibbs measure. The Boltzmann distribution for the fractional number of particles $X=N_{i} / N$ occupying a set of states i possessing energy $E_{i}$ is

$$
X=\frac{N_{i}}{N}=g_{i} \cdot \exp \left(\frac{-E_{i}}{k_{B} T}\right) / Z(T)
$$

where $k_{B}$ is the Boltzmann constant, $T$ is temperature (assumed to be a well- defined quantity), $g_{i}$ is the degeneracy (meaning, the number of states having energy $E_{i}$ ), $N$ is the total number of particles and $Z(T)$ is the partition function [2-6].

$$
\begin{gathered}
N=\sum_{i} N_{i} \\
Z(T)=\sum_{i} g_{i} \cdot \exp \left(\frac{-E_{i}}{k_{B} T}\right)
\end{gathered}
$$

Alternatively, for a single system at a well-defined temperature, it gives the probability that the system is in the specified state. The Boltzmann distribution applies only to particles at a high enough temperature and low enough density that the quantum effects can be ignored and the particles obey Maxwell-Boltzmann statistics. The Boltzmann distribution is often expressed in terms of $\beta=1 / \mathrm{kT}$ where $\beta$ is referred to as thermodynamic beta. The term "exp $\left(-\beta E_{i}\right)$ " or "exp $\left(-E_{i} /(k B T)\right) "$, which gives the (unnormalized) relative probability of a state, is called the Boltzmann factor and appears often in the study of physics and chemistry [12, 13].

The gyromagnetic ratio (also sometimes known as the magnetogyric ratio in other disciplines) of a particle or system is the ratio of its magnetic dipole moment to its angular momentum, and it is often denoted by the symbol " $\gamma$ ", gamma. Its SI units are radian per second per tesla $(\mathrm{R} /(\mathrm{S} \cdot \mathrm{T}))$ or, equivalently, coulomb per kilogram $(\mathrm{C} / \mathrm{kg})$. The term "gyromagnetic ratio" is sometimes used as a synonym for a different but closely related quantity, the g-factor. The g-factor, unlike the gyromagnetic ratio, is dimensionless. For more on the g-factor, see below, or see the article g-factor. Protons, neutrons, and many nuclei carry nuclear spin, which gives rise to a gyromagnetic ratio as above. The ratio is conventionally written in terms of the proton mass and charge, even for neutrons and for other nuclei, for the sake of simplicity and consistency. The relation is as follows:

$$
\gamma=\frac{e}{2 m_{p}} g=g \frac{\mu_{N}}{\hbar}
$$

Where $\mu_{N}$ is the nuclear magneton, and $\mathrm{g}$ is the $\mathrm{g}$ factor of the nucleon or nucleus in question. The gyromagnetic ratio of a nucleus is particularly important because of the role it plays in nuclear magnetic resonance (NMR) and magnetic resonance imaging (MRI). These procedures rely on the fact that nuclear spins press on a magnetic field at a rate called the Larmor frequency (as discussed previously), which is simply the product of the gyromagnetic ratio with the magnetic field strength. Approximate values for hydrogen atom nuclei equals $42.5787(\gamma / 2 \pi(\mathrm{MHz} / \mathrm{T}))$ [2-6]. 


\subsection{Moore's Law}

Electronic computer building is a fast improving technology but its future is yet to be determined. Gordon Moore, founder of Intel, observed an interesting rule called Moore's law in 1965. He concluded that since the invention of transistors the number of transistors per chip roughly doubled every 18-24 months (see Figure 1) [14].

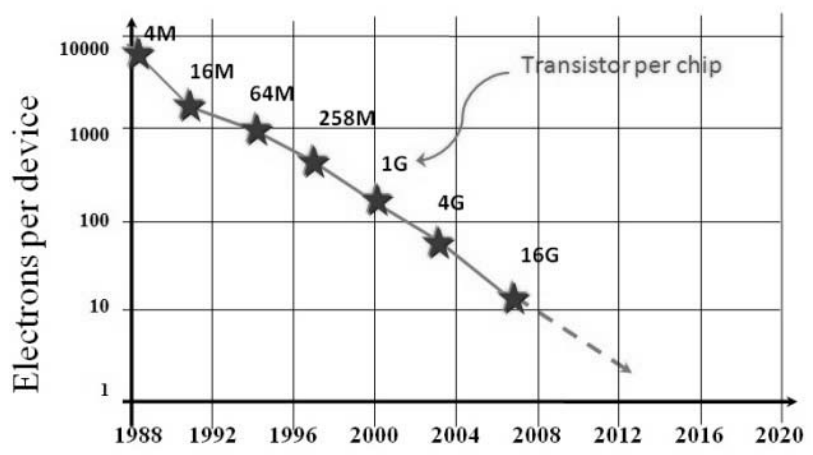

Figure 1. Representation of Moore's law. The years are given horizontally; the number of electrons per device is represented vertically.

It means an exponential increase in the computing power of computers. Although this was an empirical observation in 1965 the law seems to be valid nowadays. This law estimates serious problem around 2015 [14].

The growth in processor's performance is due to the fact that we put more transistors on the same size microchip.

This requires smaller and smaller transistors, which can be achieved if we are able to draw thinner and thinner lines onto the surface of a semiconductor disk. Around nanometer thickness we reach the nano world, where the new rules are explained by the quantum mechanics [14].

\subsection{Quantum Computer}

General purpose quantum computers do not exist yet nor are they likely to exist for 20-30 years, although small-scale laboratory models and small specialized commercial models have been developed [16]. Owing to its property of large scale integration, the superconducting qubits are the promising candidates for scalable quantum computing [17]. Nowadays the power and capability of a quantum computer is primarily theoretical speculation; the advent of the first fully functional quantum computer will undoubtedly bring many new and exciting applications [15].

Recently, much attention has been paid to the physical realization of a quantum computer, which works on the fundamental quantum mechanical principle. The quantum computer can solve certain hard problems exponentially faster than its classical counterpart. By using unitary quantum logic networks, a conventional quantum computer may be implemented [17].

On the other hand, Raussendorf and Briegel recently proposed an intriguing alternative quantum computing strategy, i.e., the one-way quantum computer, which constructs quantum logic networks by using only single qubit projective measurements on a generated cluster state. In the quantum computer, quantum information is encoded in the cluster state, processed, and read out from the cluster state. The quantum computer is universal in the sense that arbitrary unitary quantum logic networks can be carried out based on a suitable generated cluster state. Cluster states thereby serve as a universal source for quantum computers. Meanwhile, the cluster states can also be used as entanglement resources, which means that other entanglement states can be constructed from the cluster states. As mentioned above, the cluster states have special characteristics and practical applications, hence the preparations of the cluster states have been implemented by many physical systems [17].

With the progress of high-precise fabricating technique, superconducting qubits have shown their competence in quantum computing. The Josephson charge qubit and flux qubit are based on the macroscopic quantum effects on superconducting circuits. The decoherence time of superconducting qubits is not very long, but the number of quantum operations that can be completed during the coherence time is also comparable with other systems. Owing to its property of large-scale integration, the superconducting qubits are the promising candidates for scalable quantum computing [17]. 


\subsection{The Quantum Bit}

In the classical information the smallest information bearing unit is called a bit. In digital computers, the voltage between the plates of a capacitor represents a bit of information: a charged capacitor denotes bit value 1 and an uncharged capacitor bit value 0 , but in advanced computers, one bit of information can be encoded using two different polarizations of light or two different electronic states of an atom. However, if we choose an atom as a physical bit then quantum mechanics tells us that, apart from the two distinct electronic states, the atom can be also prepared in a coherent superposition of the two states. This means that the atom is both in state 0 and state 1 . Quantum computers use quantum states which can be in a superposition of many different numbers at once. The simplest quantum system can be described by means of a two-dimensional complex valued vector in a two-dimensional Hilbert space. We call it quantum bit, qubit or qbit (Figure 2). A quantum computer manipulates qubits by executing a series of quantum gates, each being unitary transformation acting on a single qubit or pair of qubits $[14,15]$.

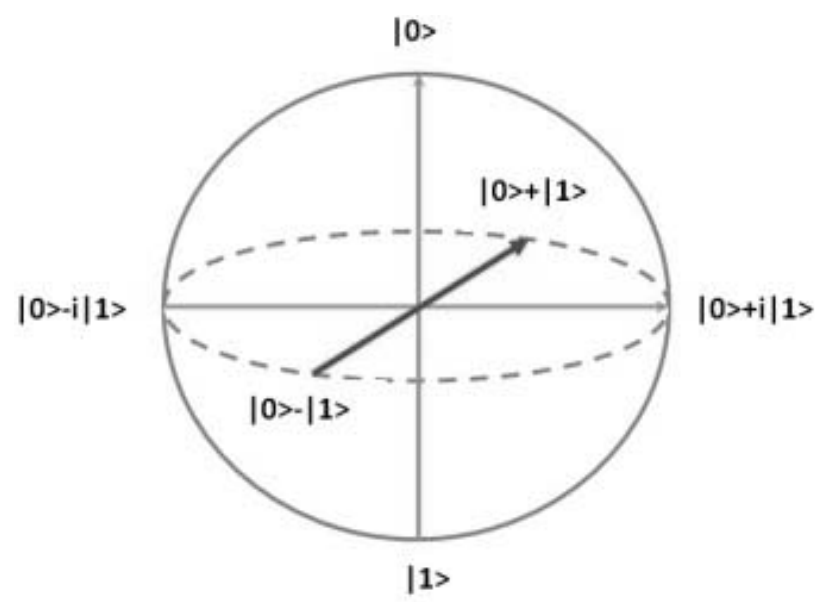

Figure 2. The general representation of a qubit in a two dimensional Hilbert-space.

In applying these gates in succession, quantum computers can perform complicated unitary transformations to a set of qubits in some initial state. The qubits can then be measured with this measurement serving as the final computational result. This similarity in calculation between a classical and quantum computer affords that in theory, classical computers can accurately simulate quantum computers. The simulation of quantum computers on classical ones is a computationally difficult problem because the correlations among quantum bits are qualitatively different from correlations among classical bits, as first explained by John Bell. For example: take a system of only a few hundred qubits, this exists in a Hilbert space of dimension that in simulation would require a classical computer to work with exponentially large matrices (to perform calculations on each individual state, which is also represented as a matrix), meaning it would take an exponentially longer time than even with a primitive quantum computer [10,11].

The simplest quantum system is a half-state of the two-level spin. Its basic states, spin-down $|\downarrow\rangle$ and spinup $|\uparrow\rangle$, may be relabelled to represent binary zero and one, i.e., $|0\rangle$ and $|1\rangle$, respectively. The state of such a single particle is described by the following wave function:

$$
\psi=\alpha|0\rangle+\beta|1\rangle
$$

The squares of the complex coefficients $|\alpha| 2$ and $|\beta| 2$ represent the probabilities of finding the particle in the corresponding states.

Generalizing this statement to a set of $k$ spin $1 / 2$ particles, we find that there are now $2 \mathrm{k}$ basis states (quantum mechanical vectors that span a Hilbert space) which is like saying that there are $2 \mathrm{k}$ possible bit-strings of length $\mathrm{k}$.

However, observing the system would cause it to collapse into a single quantum state corresponding to a single answer a single list of $5001 \mathrm{~s}$ and $0 \mathrm{~s}$, as dictated by the measurement axiom of quantum mechanics. The reason for this is an exciting result derived from the massive quantum parallelism achieved through superposition, which would be the equivalent of performing the same operation on a classical super-computer with 10150 separate processors [10]. 


\section{An introduction to binary logics and applications}

Digital design is concerned with the design of digital electronic circuits. The subject is also known by other names such as logic design, switching circuits, digital logic, and digital systems. Digital circuits are employed in the design of systems such as digital computers, electronic calculators, digital control devices, digital communication equipment, and many other applications that require electronic digital hardware [14].

\subsection{Decoder}

Discrete quantities of information are represented in digital system with binary codes. A binary code of $n$ bits is capable of representing up to distinct elements of the coded information. A decoder is a combinational circuit that converts binary information from $n$ input lines to a maximum of $2^{n}$ unique output lines. If the $n$-bit decoded information has unused or don't-care combinations, the decoder output will have less then $2^{n}$ outputs [18].

As an example, consider the 3-to-8 line decoder circuit of Figure 3.

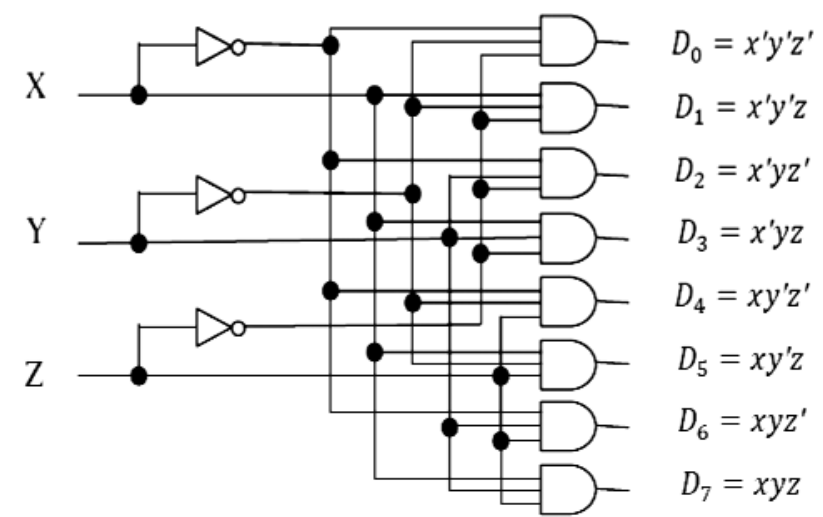

Figure 3. 3-to-8 line decoder.

The three minterms are decoded into eight outputs, each output representing one of the minterms of the 3-input variables. The three inverters provide the complement of the inputs, and each one of the eight AND gates generate one of the minterms. A particular application of this decoder would be a binary-to octal conversion.

The input variables may represent a binary number, and the outputs will then represent the eight digits in the octal number system. However, the 3-to-8 line decoder can be used for decoding any 3-bit code to provide eight outputs, one for each element of the code [18].

\begin{tabular}{ccc|cccccccc}
\hline \multicolumn{2}{c|}{ Input } & \multicolumn{1}{c}{ Output } \\
$\mathbf{X}$ & $\mathbf{Y}$ & $\mathbf{Z}$ & $\mathbf{D}_{\mathbf{0}}$ & $\mathbf{D}_{\mathbf{1}}$ & $\mathbf{D}_{\mathbf{2}}$ & $\mathbf{D}_{\mathbf{3}}$ & $\mathbf{D}_{\mathbf{4}}$ & $\mathbf{D}_{\mathbf{5}}$ & $\mathbf{D}_{\mathbf{6}}$ & $\mathbf{D}_{\mathbf{7}}$ \\
\hline 0 & 0 & 0 & 1 & 0 & 0 & 0 & 0 & 0 & 0 & 0 \\
\hline 0 & 0 & 1 & 0 & 1 & 0 & 0 & 0 & 0 & 0 & 0 \\
\hline 0 & 1 & 0 & 0 & 0 & 1 & 0 & 0 & 0 & 0 & 0 \\
\hline 0 & 1 & 1 & 0 & 0 & 0 & 1 & 0 & 0 & 0 & 0 \\
\hline 1 & 0 & 0 & 0 & 0 & 0 & 0 & 1 & 0 & 0 & 0 \\
\hline 1 & 0 & 1 & 0 & 0 & 0 & 0 & 0 & 1 & 0 & 0 \\
\hline 1 & 1 & 0 & 0 & 0 & 0 & 0 & 0 & 0 & 1 & 0 \\
\hline 1 & 1 & 1 & 0 & 0 & 0 & 0 & 0 & 0 & 0 & 1 \\
\hline
\end{tabular}

Table 1. Truth table of 3-to-8 line decoder.

The operation of the decoder may be further clarified from its input-output relationships, listed in Table 1. Observe that the output variables are mutually exclusive because only one output can be equal to 1 and represents the minterm equivalent of the binary number presently available in the input lines [18].

\subsection{Encoder}

An encoder is a digital function that produces a reverse operation from that of a decoder. An encoder has $2^{n}$ (or less) input lines and $n$ output lines. The output lines generate the binary code for $2^{n}$ input variables. An example of an encoder is shown in Figure 4 [18].

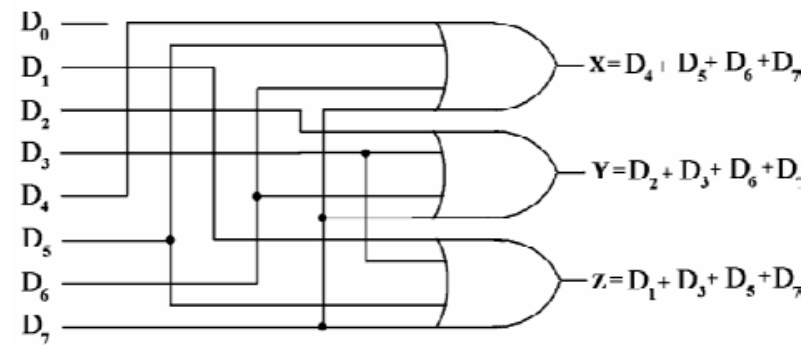

Figure 4. Octal-to-binary encoder. 
The octal-to-binary encoder consists of eight inputs, one for each of the eight digits, and three outputs that generate the corresponding binary number. It is constructed with OR gates whose inputs can be determined from the truth table given in Table 2. The low-order output bit $z$ is 1 if the input octal digit is odd. Output $y$ is 1 for octal digits $2,3,6$, or 7 . Output $x$ is a 1 for octal digital $4,5,6$, or 7 . Note that $D_{0}$ is not connected to any OR gate; the binary output must be all 0's. This discrepancy can be resolved by providing one more output to indicate the fact that all inputs are not 0's [18].

\begin{tabular}{cccccccc|ccc}
\hline \multicolumn{1}{c|}{ Input } & \multicolumn{3}{c}{ Output } \\
$\mathbf{D}_{\mathbf{0}}$ & $\mathbf{D}_{\mathbf{1}}$ & $\mathbf{D}_{\mathbf{2}}$ & $\mathbf{D}_{\mathbf{3}}$ & $\mathbf{D}_{\mathbf{4}}$ & $\mathbf{D}_{\mathbf{5}}$ & $\mathbf{D}_{\mathbf{6}}$ & $\mathbf{D}_{\mathbf{7}}$ & $\mathbf{X}$ & $\mathbf{Y}$ & $\mathbf{Z}$ \\
\hline 1 & 0 & 0 & 0 & 0 & 0 & 0 & 0 & 0 & 0 & 0 \\
\hline 0 & 1 & 0 & 0 & 0 & 0 & 0 & 0 & 0 & 0 & 1 \\
\hline 0 & 0 & 1 & 0 & 0 & 0 & 0 & 0 & 0 & 1 & 0 \\
\hline 0 & 0 & 0 & 1 & 0 & 0 & 0 & 0 & 0 & 1 & 1 \\
\hline 0 & 0 & 0 & 0 & 1 & 0 & 0 & 0 & 1 & 0 & 0 \\
\hline 0 & 0 & 0 & 0 & 0 & 1 & 0 & 0 & 1 & 0 & 1 \\
\hline 0 & 0 & 0 & 0 & 0 & 0 & 1 & 0 & 1 & 1 & 0 \\
\hline 0 & 0 & 0 & 0 & 0 & 0 & 0 & 1 & 1 & 1 & 1 \\
\hline
\end{tabular}

Table 2. Truth table of octal-to-binary encoder.

The encoder in Figure 4 assumes that only one input line can be equal to 1 at any time; otherwise the circuit has no meaning. Note that the circuit has eight inputs and could have $28=256$ possible input combinations. Only eight of these combinations have any meaning. The other input combinations are don't-care conditions [14].

Encoders of this type (Figure 4) are not available in IC packages, since they can be easily constructed with OR. The type of encoder available in IC form is called a priority encoder. These encoders establish an input priority to ensure that only the highest-priority input line is encoded. Thus, in Table 2, if priority is given to an input with higher subscript number over one with a lower subscript number, then if both $D_{2}$ and $D_{5}$ are logic-1 simultaneously, the output will be 101 because $D_{5}$ has a higher priority over $\mathrm{D}_{2}$. Of course, the truth table of a priority encoder is different from the one in Table 2 [14].

\section{Qubit and quantum decoder design}

In this section we propose a new method for qubit and decoder implementation using the quantum theory. We use the states of hydrogen nuclear spin.

\subsection{Hydrogen Nuclear Spin}

The reason statistics of the Maxwell-Boltzmann probability distribution function is used in order to do this is a direct result of the infinite small size of atoms, molecules and spin populations. If a computer were to keep track of a sample of the nuclear spins at the selected temperature and pressure, it would need to dynamically account for the position and velocity vectors for the number of nuclear spins (here, for hydrogen atoms). This is too many operations for most modern computers to handle adequately. Other problems occur of course which stem from quantum mechanics and our increasing inability to precisely know the exact positions and velocities if nuclear spins were chosen to examine [19].

The values of the magnetic field powerful (B0, in Tesla), Frequency ( $U$ in $\mathrm{MHz})$, Boltzmann distribution ratio $\left(X=N_{i} / N\right)$ and $T=\ln (1 /(1-X))$ of hydrogen nuclear magnetic resonance are shown in Table 3. The "T" values which are shown in Table 3 introduce the Napierian logarithmic of the ratio of $1 /(1-X)$ as a digital index (constant temperature).

In this study, the values that are introduced in Table 3 were utilized as input for a decoder model. The first value of the magnetic field was approximated to zero. The Boltzmann distribution ratio $\left(X=N_{i} / N\right)$ and the "T" values decreased by increasing the magnetic field ( $B_{0}$, in Tesla) and the induced frequency. By using these 3 values in the decoder model of this study, different outputs can be observed during the process.

Figure 7 shows the process of input of the different 3 values for $\mathrm{H}$-atom ( $\mathrm{B}_{0}, \mathrm{U}$ and $\mathrm{Y}$ values) and various matrices output as result of the process. By changing one or more of the values, various matrices result as output.

\subsection{Qubit Design}

The external field magnitudes on the nuclear spin produce the spin moment in the Larmor frequency.

If this frequency overlaps by electromagnetic radiation with the Larmor frequency, it gives the energy and change of spin state (see Figure 5). 
The previously mentioned practice is the act of writing. By removing the external field, the spin returns to an earlier state which is called the act of reading. Here the effect of reading is radiated in the wave form.

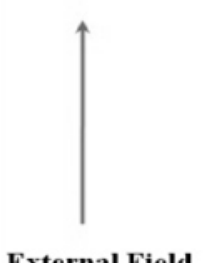

External Field

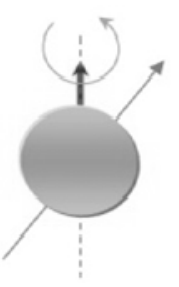

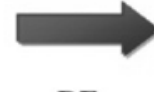

RF

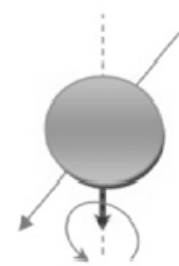

Absorption

Figure 5. Nuclear spin in external field.

Considering Table 1 and the above materials, $\mathrm{B}_{0}$ is drawn on as a logic zero:

$0 \quad$ (Low): $\quad B_{0}=10^{-5} T, \quad f_{1}=4.25787 \times 10-4 \quad M H Z$, $\mathrm{T}_{1}=23.409663$.

And $B_{1}=2 T$ that makes $X$ decrease, called the binary one:

$1 \quad$ (High): $\quad B_{1}=2 T, \quad f_{2}=85.157444 \quad M H Z$, $\mathrm{T} 2=11.203597$.

In this design we can use some $B$ for the zero and one state that shows the relation between $B$ and $T$ in Figure 6.

\begin{tabular}{ccccc}
\hline & $\begin{array}{c}\text { Magnet } \\
\text { ic Field } \\
\left(\boldsymbol{B}_{\boldsymbol{0}}, \text { in }\right. \\
\text { Tesla) }\end{array}$ & $\begin{array}{c}\text { Frequency } \\
(\boldsymbol{U} \text { in MHz) }\end{array}$ & $\begin{array}{c}\text { Boltzmann } \\
\text { Distribution Ratio }\end{array}$ & $\begin{array}{c}\boldsymbol{\tau}= \\
\mathbf{I n}(\mathbf{1} / \mathbf{1}-\mathbf{X})\end{array}$ \\
\hline $\mathbf{1}$ & $10^{-5} \approx 0$ & $\begin{array}{c}4.25787 \times \\
10^{-4}\end{array}$ & 0.999999999931874 & 23.409663 \\
\hline $\mathbf{2}$ & 0.5 & 21.289361 & 0.999996593708047 & 12.589886 \\
\hline $\mathbf{3}$ & 1.0 & 42.578722 & 0.999993187427698 & 11.896741 \\
\hline $\mathbf{4}$ & 1.5 & 63.868083 & 0.999989781158951 & 11.491277 \\
\hline $\mathbf{5}$ & 2.0 & 85.157444 & 0.999986374901806 & 11.203597 \\
\hline $\mathbf{6}$ & 2.5 & 106.446805 & 0.999982968656265 & 10.980455 \\
\hline $\mathbf{7}$ & 3.0 & 127.736166 & 0.999979562422326 & 10.798135 \\
\hline $\mathbf{8}$ & 3.5 & 149.025527 & 0.999976156199990 & 10.643986 \\
\hline $\mathbf{9}$ & 4.0 & 170.314888 & 0.999972749989256 & 10.510457 \\
\hline & & & & \\
\hline
\end{tabular}

Table 3. The spin status of hydrogen in different magnetic fields.
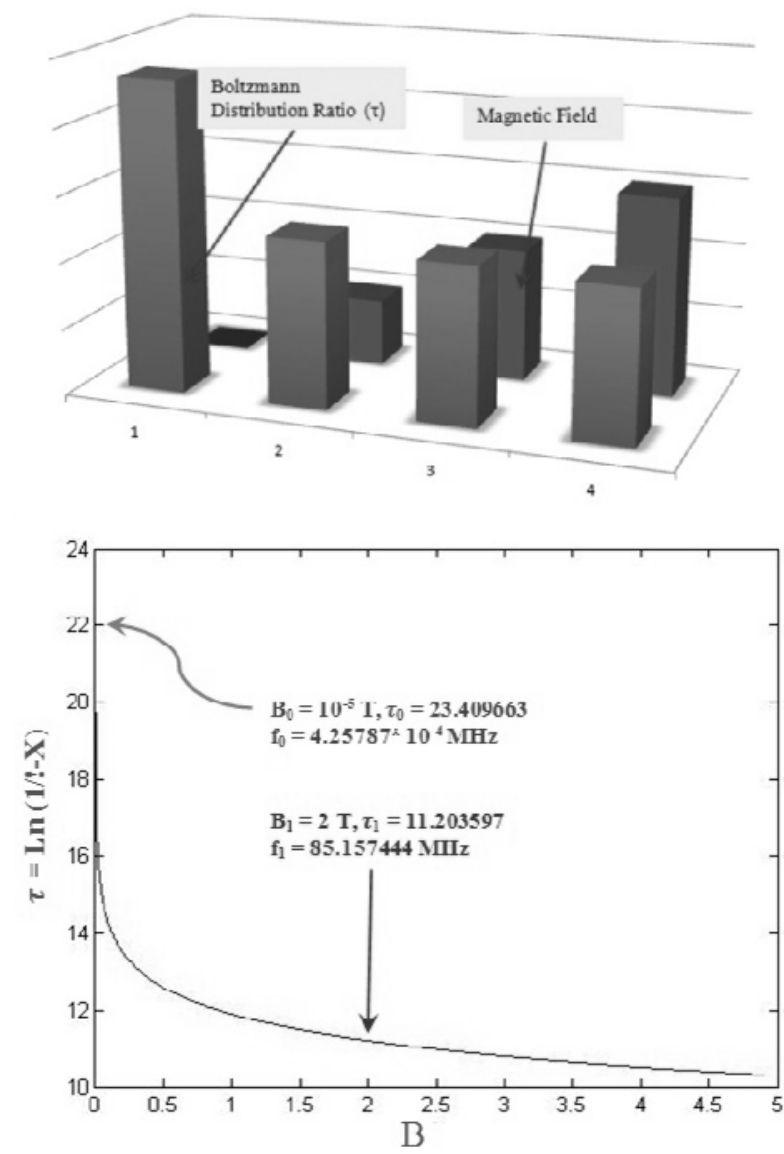

Figure 6 . The relation between $\mathrm{B}$ and $\mathrm{T}$.

By implementing the special frequency in the external field effect use writes in the bit in order to release the energy in atom for the read in the bit.

\subsection{Quantum decoder design}

In the excitation process of an organic compound, if there is frequency sweeping in excitation, atoms of hydrogen resonate in separated frequencies. Different positions of hydrogen atoms is the reason for this phenomenon. We can use this phenomenon implementation as in Figure 7. 


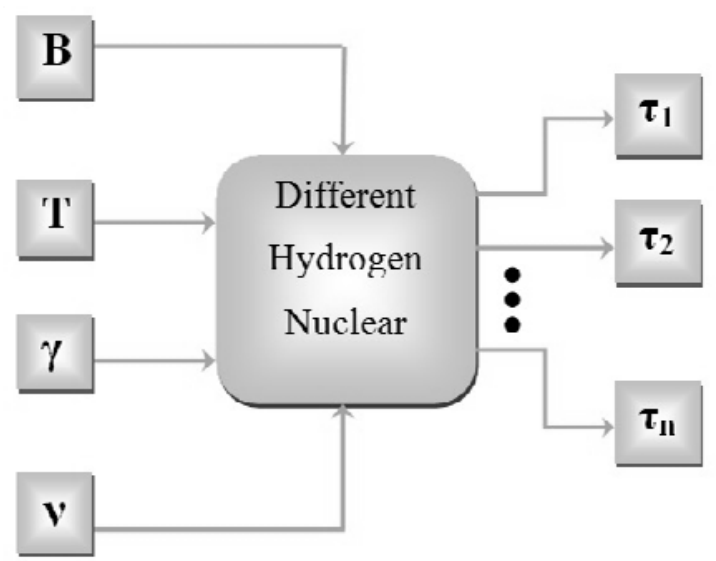

Figure 7. Decoder implementation.

Where $B$ is external field magnitude, $v$ is the Larmor frequency of the field, $T$ is temperature and $Y$ is magnetogyric of atoms. $T_{i}$ is yield from Eq. (11).

$$
\tau=\operatorname{Ln}\left(\frac{1}{1-X}\right)
$$

We will have a unique peak in one of the $f_{1}, f_{2} \ldots f_{n}$ frequencies. If we have a peak in $f_{i}$, then associated output $T_{i}$ is high. Thus, it can be used as a decoder. In a similar manner. It can be used for multi true value logic (as J. Lukasiewicz logic) implementation.

\section{Conclusion}

This paper introduces the concept of a new method for the design, analysis, modeling, simulation of a qubit and decoder in a quantum computer. Based on our proposal, we can summarize our findings as follows:

1. We used the spin field effect for the qubit design. The difference between the classical bit and the qubit proposed is that a qubit can be in a state other than 0 or 1 . Additionally, the decoder proposed has several lines for the selected in output on a scale of the other one.

2. The suggested qubit and decoder needs to be verified by more theoretical and experimental research to determine its potential as an applicable feature in the future.
3. The authors believe that the proposed techniques can be applied to quantum computing. Further research could be conducted to confirm the effectiveness of the proposed techniques using a variety of quantum computing techniques.

\section{References}

[1] V. G. Cattaneo, A. Leporati and R. Leporini, Quantum Conservative Many-valued Computing, Fuzzy Sets and Systems, Elsevier, no. 159, September. 2007, pp. 10011030.

[2] J. Keeler, Understanding NMR spectroscopy, Wiley, 2006.

[3] W. H. Louisell, Quantum Statistical Properties of Radiation, John Wiley \& Sons, 1973.

[4] I. N. Levine, Quantum Chemistry, 4th edition, Prentice-Hall Interna-tional Inc, New York, 1991.

[5] M. A. Bernstein, K. F. King and X. J. Zhou. Handbook of MRI Pulse Sequences. San Diego: Elsevier Academic Press, 2004.

[6] R. C. Weast, M. J. Astle. Handbook of chemistry and physics. Boca Raton: CRC Press. p. E66. ISBN 0-84930463-6, ed 1982.

[7] X. Zheng, P. Dong, Z. Xue, Z. Cao, Generation of Cluster States with Josephson Charge Qubits, Physics Letters A, Elsevier, no. 356, January. 2007, pp. 156-160.

[8] W. Heisenberg, Über den anschulichen Inhalt der quantentheo-retischen kinematik und Mechanik, Z. Phys. 43, 172-198 (1927).

[9] E. D. Becker, High Resolution NMR, Academic Press, 23, 1969 .

[10] M. H. Levitt. Spin Dynamics, Jon Wiley and Sons, 196, 2001.

[11] M. Munowitz, Coherence and NMR, John Wiley \&Sons, 18, 1988.

[12] Carter, Ashley $\mathrm{H}$, Classical and Statistical Thermodynamics, Prentice -Hall, Inc, New Jersey, 2001.

[13] Pathria, R. K, Statistical Mechanics, ButterworthHeinemann, 1996. 
[14] L. Bacsardi, Satellite Communication Over Quantum Channel, Acta Astronautica, PERGAMON, no. 61, March. 2007, pp. 144-149.

[15] S. Imre, B. Ferenc, Quantum Computing and Communications: an engineering approach, Wiley, New York, 2005.

[16] H. Guo, J. Zhang and G. J. Koehler, A survey of quantum games, Decision Support Systems, Elsevier, no. 46 , April. 2008, pp. 318-332.

[17] X. H. Zheng, P. Dong, B. L. Yu and Z. L. Cao, Preparation of Cluster States with Superconducting Qubit Network, Solid State Communica-tions, Elsevier, no. 144, September. 2007, pp. 206-209.

[18] M. M. Mano, Digital Design, Prentice Hall, 1984, pp. $167-175$.

[19] http://user.mc.net/ buckeroo/MXDF.html 